\title{
Mayers-Scotten's 4-M Model: A Qusai-Experimental Study of Pashto-English Morphological Ability
}

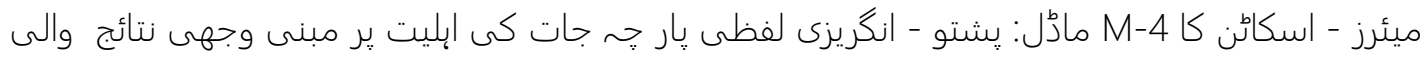 \\ تجرباتى تحقيق
}

Accepted: November 8, 2020

Written by:

Fatima-tu-Zahara ${ }^{1}$

https://orcid.org/0000-0001-5392-3149

Tahir Saleem ${ }^{2}$

https://orcid.org/0000-0002-7952-0572

Nadia Joiya ${ }^{3}$

https://orcid.org/0000-0001-6544-7039

Farhat Abdullah $^{4}$

https://orcid.org/0000-0003-0409-6794

\begin{abstract}
Bilingualism has been a myth for linguists and language policy and planning professionals for decades. Current study observes morphological ability and bilingual profile of L1 and L2 of Pashto mother-tongue children. Bilingual profile of the participants measured through bilingual language profile (BLP) tool. Both L1 and L2 children were tested before and after intervention. Morphological ability was measured through $4 \mathrm{M}$ model. After 4 weeks of intervention, experimental child was able to read and write complex words with bridge morphemes. In contrast, controlled sample was not exposed to the intervention. The participants performed assigned language tasks and their performance-expressions were analyzed. Study confirms that early and late bridge morphemes are acquired and children have intelligibility of the language despite the fact that BLP shows low profile of the mother tongue. Significant effects of mother tongue were recorded in the participants' performance. Effects of explicit morphological instruction was focused on identifying Pashto orthography and applying morphological ability on word formations. Findings reveal bilingual profile and patterns of morphological ability after didactic practice of intervention. Intervention contributed in developing Pashto orthography that was crucial for reading and writing proficiency. Direct impact on text-based inference and reading comprehension was another milestone of this
\end{abstract}

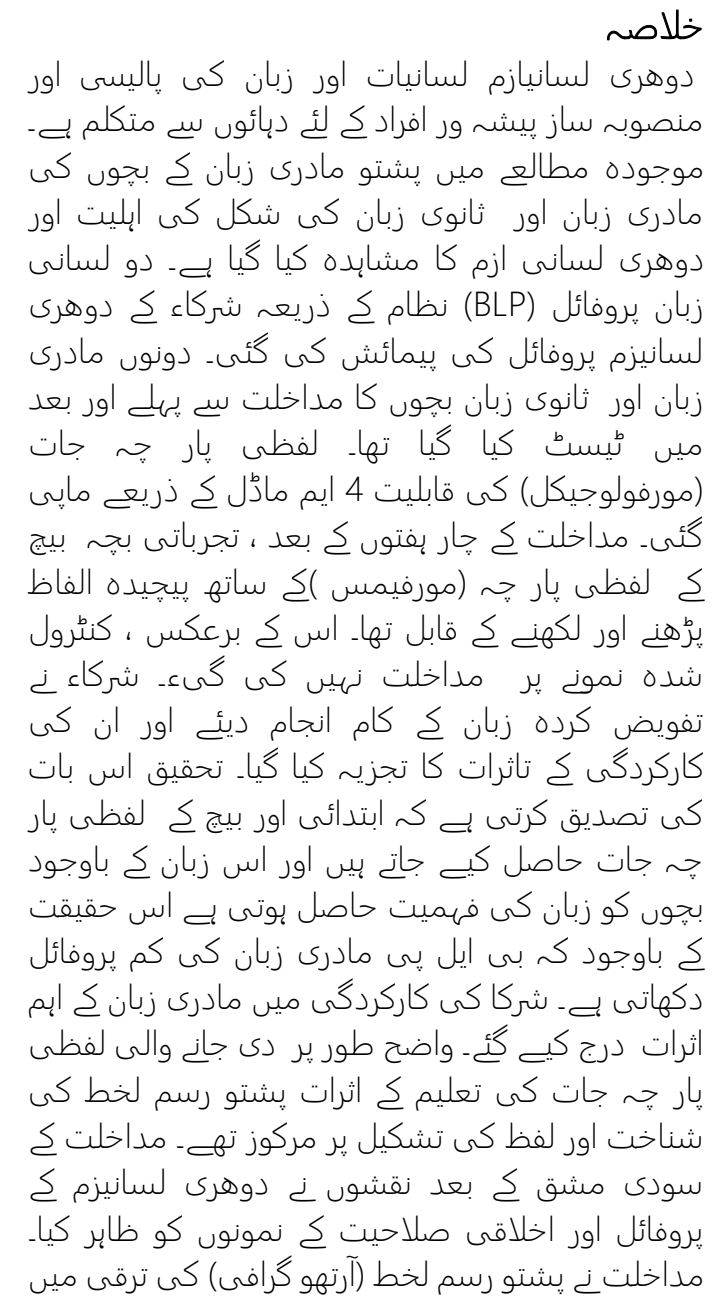

\footnotetext{
${ }^{1}$ M.Phil Applied Linguistics Scholar, Department of English, University of Central Punjab Lahore Pakistan.

${ }^{2} \mathrm{PhD}$ in Linguistics and Literature, Assist. Professor, Department of English, University of Central Punjab Lahore Pakistan.

${ }^{3}$ M.Phil Applied Linguistics Scholar, Department of English, University of Central Punjab Lahore Pakistan.

${ }^{4} \mathrm{PhD}$ in Linguistics Scholar, Lecturer in English, Department of English, University of Central Punjab Lahore Pakistan.
} 


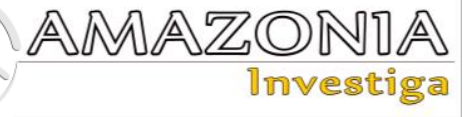

qusai-experimental research. This model can be used for longitudinal studies.

Keywords: Morphological ability, Pashto bilinguals, $4 \mathrm{M}$ model, late morphemes, bilingual education.

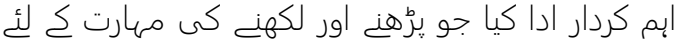

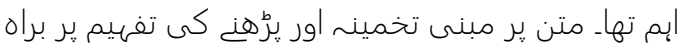

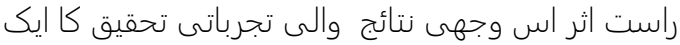

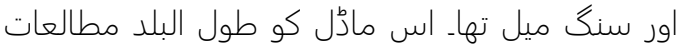

$$
\begin{aligned}
& \text { ك لئ استعمال كيا جاسكتا بـــ }
\end{aligned}
$$

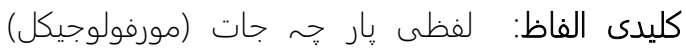

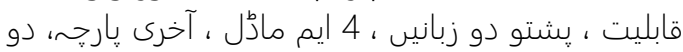

$$
\begin{aligned}
& \text { لسانى تعليم }
\end{aligned}
$$

\section{Introduction}

English and Pashto are from Indo-European family of languages and have several similarities in morphological functions and forms when it comes to inflection. Both languages have Greek influence as their proto-language. Nevertheless, Pashto stands in the category of morphologically-rich languages when compared with English (Khan et al., 2016). Eventually, cognitive faculty of human mind and the Minimalist program (MP) can further assess morphological abilities in the paradigm of Distributional Morphology (DM) (López, 2018). Given that current research and empirical data further provide evidence for a multilingual lexicon where components of each language are well adjusted for language production and comprehension (Miikkulainen \& Kiran, 2009). A previous research indicated that the acquisition of morphosyntactic features decline in adults as compared to early language learners (Bosch \& Clahsen, 2019).

This research observes Pashto and English morphemes and their inflectional morphemes through Mayers-Scotten's 4-M model. Khan et al. (2016) observed markedness on Pashto morphemes. As Pashto common nouns are inflected for number, gender and case and both languages use inflection for irregular nouns as well. Similarly, English possessive and proper nouns are inflected in gender regarding vocative case. Moreover, the influence of Greek in Pashto gender marking is also observed.

As has been discussed, bilingual profile can show overlapping of languages in language use and language proficiency. The current study intends to examine the effects of this overlapping on morphological ability of young learners. Furthermore, the study aims to better understand why bilingual profile show different levels of frequencies for both native language and second language. Consequent exposure to L2 may lead to low level of proficiency of L1, regardless of its use and learners' attitudes towards their mother tongue.
Further, bilingualism and language maintenance positively affect morphological ability. Bilinguals with a positive attitude have greater morphological ability. As mention earlier, Pashto is a rich morphological, less resourced language that lends itself to further analysis/comparison with other languages, expands its vocabulary through code mixing and accepts patterns from other languages. Motivation was to determine pattern of inflectional and derivational values of dominant (English) and less dominant (Pashto) language, to add findings of this study into existing literature or device language teaching modules. This experimental research is a followup study of 4M model constraints by (Khan et al., 2016) and yet another valid measure of morphological ability as compared to the other influential studies with two different measures. It further establishes existing scales and provides evidence for future research. Children acquire past tense morphemes through analogy with other words in their vocabularies as several studies confirm this notion of language acquisition (Nicoladis \& Marentette, 2007).

In this regard, pilot studies with systematic pedagogical interventions can be very helpful in expanding research in this area. Feasibility, duration, adverse contexts and costs can be evaluated through pilot study, prior to the fullscale inquiry. Further, implications are likely to be realized in empirical research, based on asymmetrical characteristics of both languages. Previous research (Khan et al., 2016) was conducted with adult learners and this research has filled the gap through involving early year learners. Language acquisition is also predicted here through both controlled and experimental participants who have acquired four types of morphemes through home-based communications. Mother-tongue was not exposed to the individuals in academic or social settings other than maternal family. Hence, present research is contributing through providing empirical evidence on two 
typologically different languages in bilingual contacts with a focus on predictors of morphological ability while ignoring individual difference.

\section{Review of Literature}

Using two or more languages everyday can cause cross-linguistic effects and there could be internal and external factors (context of exposure) of this language contact phenomenon (Chondrogianni \& Vasić, 2016). BoussofaraOmar (2013) studied Arabic diglossic conditions with reference to Matrix Language Frame (MLF) and refined his evidence through 4-M model and Abstract Level model (ALM). These models are used extensively for identifying morphological abilities and grammars in contact languages. Previous researches (Hsu et al., 2019; Snyder et al., 2008) on morphological ability were limited to bilingual profile and little attention has been paid to identifying late bridge or outsider system morphemes. This, therefore is the focus of the current research. Pakistan is a multilingual and multicultural country where English is functioning as a Lingua France (Coleman, 2010). Though English and Urdu are the languages of educational systems, Pashto is well maintained by its speakers and this positive attitude has given rise to its extensive usage on social media chats with a Pashto-English variety. Nicoladis et al. (2007) observed same phenomenon of language maintenance where young learners use language with complex morphological patterns when introduced to their L1 (Rock, 2018; Tomasello, 2004). Another study by Ocal and Ehri (2017) proposed that vocabulary items are correlated with morphological patterns and can be a considerable success predictor.

Though it is hard to find any research on Pashto intervention, probing participants about their reading and writing engagement, two intervention studies (Khan et al., 2016; Beaudrie, 2017) draw our attention towards morphological ability of Pashto speakers. Likewise, Bowles (2018) identified three pedagogical intervention based research initiatives for Spanish. Another study observed narrative abilities of bilinguals over time (Parra et al., 2018). These interventions pose another problem to the researchers: If participants need more exposure to reading and writing as compared to listening and speaking, then, to what extent does these activities guarantee improvement?
Given that Kecskes (2000) observed dynamic process of multilingual development where language transfer is different and underlying foundation is same through constant language interaction (Kecskes \& Papp, 2000). In terms of language acquisition, multilinguals have an edge on monolinguals when linguistic knowledge is compared (van Hooff, 2018). These findings further reveal complexity of grammars in bilingual and multilingual contacts where changing nature of grammars and evolving MLF becomes challenging. Monolingual grammatical frame can be understood through comparison between languages in bilinguals (Vihman, 2018). Similar studies confirm that multiple orthographies are responsible for increasing multilingual proficiencies (Abu-Rabia \& Sanitsky, 2010; Soudi et al., 2007).

Khan and Khalid (2018) documented asymmetrical structure of Pashto-English data while considering Pashto as matrix language and English as embedded language. Pashto is considered responsible for well-formedness of the language expressions produced by the participants where late system morphemes are reportedly triggered to support the structure. Current study investigates morphological ability of Pashto-English bilinguals based on their bilingual profile, which is addressing the limitations of previous research initiatives on adult learners. The study further documented the diverse morphological patterns through 4-M model, identifying effects of embedded language on matrix language. For investing the current phenomenon, this research proposed the following research questions:

1. How do bilingualism and language dominance positively affect morphological ability?

2. How do language in history, language in use and language proficiency determine bilingualism?

3. To what extent are early year learners able to acquire early and late system morphemes?

\section{Methodology}

The current study was conducted in a homebased Pakistani context amid Covid-19. PashtoEnglish communication was collected and analysed through digital mediums without any involvement from commercial sector or any conflict of interest. For qusai-experiment, two Pashto mother tongue children were selected in the age range of (4-6). English was medium of instruction in their schools. Bilingual profile of 14 adult Pashto speakers was also measured 


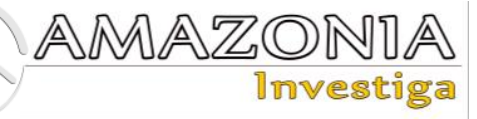

through BLP. In the first place, BLP tool was used to analyse data quantitatively. BLP was selected because of its convenient design and accuracy reported in several studies (Gertken \& Birdsong, 2014). BLP was collected through online google forms whereas speech samples and voice messages were collected, recorded and analyzed through google drive and WhatsApp. BLP was tested before sharing with the participants. After successful testing, extra responses were discarded by the researcher. Language dominance was determined through careful analysis of all the responses in BLP report. All participants used BLP for selfassessment.

After this quantitative analysis, 4-M model was used to identify morphological ability of Pashto mother tongue early year learner. After pre-test, an intervention was introduced to the experimental child. Experimental participant was observed for the changes in morphological ability. All 4 types of $4 \mathrm{M}$ model were investigated. Experimental child received designated treatment and then administered accordingly. Reliability was achieved through involvement of two raters at both pretest and posttest level. Individual differences are an intervening variable that is related to dependent variable (Language Task score), whereas, independent variables are controlled through language dominance score in BLP scale (Birdsong et al., 2012). Internal and external validity is achieved through triangulation. Qualitative research, intervention and $4 \mathrm{M}$ model exposed underlying theory and nature of the language development in natural experiment.

\section{Results and Discussion}

Language dominance and profile were calculated for all 14 participants whereas 2 participants contributed in experimental comparison. Language profile and dominance score as automatically generated from self-assessment of online forms and data was extracted from BLP (Birdsong, 2012). BLP was adapted for PashtoEnglish bilinguals. Bilingual profiles confirm low level of the mother tongue as there was no trace of reading and writing ability. Same is the case with adult Pashto-English bilinguals. Language dominance score was identified through frequency measures.

Table 1.

Bilingual profile and language dominance.

\begin{tabular}{llllc}
\hline Language & $\begin{array}{l}\text { Language } \\
\text { History }\end{array}$ & Language use & $\begin{array}{l}\text { Language } \\
\text { proficiency }\end{array}$ & Language attitudes \\
\hline ENGLISH & 17.706 & 10.9 & 45.4 & 13.62 \\
PASHTO & 27.24 & 19.62 & 29.51 & 47.67 \\
\hline
\end{tabular}

Findings show patterns of both languages and data is aligned with individual exposure to the language. Language history shows high frequency of the native language but the difference is found in language proficiency where self-assessment shows higher level of English than Pashto. Though Pashto is more in use and is strongly backed by Pashto speakers, this aspect is confirmed through attitude score, it has not affected its proficiency. All three categories of BLP confirmed low level of the mother tongue as compared to the high level of the L2.

For the second step of analysis, morphological ability of experimental child was observed through repeated language tasks. Myers-Scotton ML model (2006), 4-M model of morphological ability was considered for identifying development of all four types of morphemes. Finally, four types of morphemes were identified in both experimental and controlled participants. Two of the morphemes are identified to be activated conceptually as indicated through the speaker's intentions: Content morphemes and early system morphemes like noun number marking. verbs, nouns, and adjectives, minus their gender, person, and number affixes, for example, are content morphemes. Content morphemes are easy to identify whereas other three types of system morphemes develop gradually. The other two are said to be activated structurally through the grammar of the ML: the late system morphemes (Myers-Scotton 2006; Myers-Scotton \& Jake, 2000). 
Table 2.

Pretest Morphological ability of experimental participant.

\begin{tabular}{|c|c|c|c|c|}
\hline Language & Content & Early & Late bridge & Late outsider \\
\hline Pashto & قلم & قلمونه & د سار ا قلم & قلم ليكي \\
\hline English & Dog & Dogs & Sara's dog & Gallops \\
\hline Pashto -English & Trout & trout- $\alpha: n$ & Da chachi trout & Da chachi trout lamboo \\
\hline
\end{tabular}

Pretest Morphological ability of controlled participant

\begin{tabular}{|c|c|c|c|c|}
\hline Language & Content & Early & Late bridge & Late outsider \\
\hline Pashto & جاجִى & جاجي & د جاجى كور & جاجى ليكي \\
\hline English & Book & Books & Mother's book & Writes \\
\hline Pashto-English & Laptop & Laptopoona & Da Fatima laptop & Laptop ruk sho \\
\hline
\end{tabular}

Other examples of words (Nouns, Verbs, PashtoEnglish compounds) include TV-gaan, Da Salma Tv and TV maat sho. Similarly, using kitaabz (books) is another example of such grammar integration (Ali Khan \& Muysken, 2014). Another morpheme 'trout' has been one of the major source of income in these areas. Local community depend on trout farming and tourists and the word 'trout' does not contain any sign of inflection, but somehow Pashto plural marking $\alpha: n$ as trout- $\alpha: n$ makes it a common practice for Pashto speakers (Ali Khan \& Muysken, 2014). Raw data of other grammatical categories was discarded as major focus of the research was on noun and verb inflections. Frequency of PashtoEnglish compounds is high in personal messages of the targeted individual. However, everyday speech samples have less content of such formations.
Both English and Pashto are from a different language family. However, Pashto orthography is similar to Arabic and Urdu and this is an extra advantage to these children. Urdu being the national language of Pakistan and Arabic being an essential language for Muslims, both have significant impact on Pashto morphological ability. When it comes to the three types of system morphemes, it is observed that an early system morpheme modifies the meaning of a single content morpheme within the immediate maximal projection (immediate phrasal unit) of a content morpheme, for example within a Noun Phrase (NP). Bridge morphemes connect two constituents (e.g., of in 'age of the child') and late outsider morphemes depend on information external to their own constituent to determine their form, with prime examples, being verb agreement (governed by the subject), object case (governed by the verb) or noun case governed by certain adpositions.

Table 3.

Posttest Morphological ability.

\begin{tabular}{|c|c|c|c|c|}
\hline Language & Content & Early & Late bridge & Late outsider \\
\hline \multirow{3}{*}{ Pashto } & Peesho & Peeshogaaney & Zama peesho & Peesho pey ski \\
\hline & Khor & Khwendey & Da Ayesha khor & Khor jarri \\
\hline & War & Waroona & Da kor war & War birta sho \\
\hline English & Building & Buildings & $\begin{array}{l}\text { Government's } \\
\text { buildings }\end{array}$ & Building collapses \\
\hline Pashto -English & Hospital & Hospitaloona & Da dolty hospital & Hospital band sho \\
\hline
\end{tabular}

The 4M model predicts that late outsider system morphemes only come from the ML (MyersScotton \& Jake, 2000, 2017). The data is compatible with the Myers-Scotton ML model (2006), with the Proviso that in Pashto itself the construction does not involve two verbs. Pashto speakers have nativized English elements for their local use and it can be considered as another example of code-mixing. (Saddiqa, 2018).

Kecskes (2006) identified loans and borrowings of code-switching between multiple languages. However, there is also a significant parallel between nouns and verbs. The English plural 


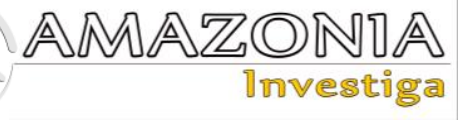

marking $-\mathrm{s}$ is sometimes lacking and sometimes redundantly present, where English itself would not have plural. It thus seems to be a primarily lexical and not grammatical feature of mixed

\section{Intervention Profile}

\begin{tabular}{lll}
\hline Phases & Activities & Weeks (1-4) \\
\hline $\mathbf{1}$ & Reading comprehension & Q and A \\
$\mathbf{2}$ & Translations( English to Pashto) & Listen and write \\
$\mathbf{3}$ & Listen and write & Practice system morphemes \\
$\mathbf{4}$ & Read and write (answer questions) & Practice system morphemes \\
\hline
\end{tabular}

Figure 1. Intervention Profile

Intervention analysis shows that Pashto orthography is very much similar to Urdu and experimental child was successful in developing reading and writing ability in just 4 weeks of the study. All four types of morphemes were already acquired through listening and speaking with mother and maternal relatives. Following activities were offered in learning sessions:

- For reading comprehension, images were shown to the participants and participants were instructed to write down words for that image with an equal chance of performance whether it is in their background knowledge or not.

- for translation, participants were supposed to read words from smart phone application and write down their corresponding words in pashto.

- for listening activities, participants were exposed to system morphemes.

- for question and answer sessions, system morphemes were used.

Diverse experiences of bilinguals are globally reported and it is often considered that L1 is spoken at home is the one that is the medium of instruction in schools, exposed in media, printed on advertisements and exercised in social events (Dunn \& Tree, 2009). However, this is not the case of several post-colonial regions of the world. Here, English has gained the status of second language moving forward from foreignlanguage-status. Second language is enjoying privilege and prestige and has an extensive usage in almost all forms of communication. A very effective way of killing a language is to deny it any place in the education system (Coleman, 2010). System morphemes were observed and stem-selection variability was identified. Language interaction and their complex morphological integration were observed during code mixing/switching. In addition to this,
Pashto-English bilingual speech. Apart from that, there are no other functional nominal elements from English present in the utterances cited, except in a few fixed expressions. presence or absence of bilingual education was observed to identify language acquisition patterns of both languages. This analysis can be compared with adult bilinguals. Data of current research deviates from dominant language hypothesis as grammatical morphemes are mixed from weaker (Pashto) to the dominant (English) and this deviation is reported in several other studies such as Keshavarz (2007). Another research on SLA suggested that monolinguals becoming multilinguals integrate both languages and there is continuous collaboration between these multiple knowledge systems (Schmid \& Köpke, 2017).

Nevertheless, findings of BLP confirmed marginalization of the native language in comparison with the second language that has more exposure in educational and professional settings. Table 1 shows bilingual profile of both languages. In the first place, Pashto stood dominant in language history. Pashto maintained its position in other two categories, language in use and language attitudes. However, in the third category, language proficiency, it was observed that participants confirmed low level of proficiency in their native language as it has less exposure in educational system. Pretest of the morphological ability confirms low proficiency level of reading and writing in Pashto. At the same time, it was observed that target child was able to comprehend all four types of morphemes after explicit instructions on morphological awareness. The speaker was able to participate in communications and translate wherever possible. All observations and audio recordings are aligned with the data. Comprehension, inferencing and morphological series of one month helped the target individual in identifying morphemes and orthography of the native language. Findings confirm direct impact on word recognition but for higher-order skills, a longitudinal study is suggested. Reading 
comprehension can be fine-tuned for global and local contextual understanding but morphological awareness is linked with orthography. It is important to note that vocabulary, phonics and grammar of the language is already in the lexicon of the child. Another research indicates that language similarity to L1 and L2 is evident in balanced bilinguals with native-like proficiency (ItaniAdams et al., 2017; Nunn \& Adamson, 2009). Intervention was tested on a few individuals prior to the post test. Several smart phone apps were reviewed and assessed by relevant PashtoEnglish teachers. Once consistency and reliability was confirmed, pretest was administered. After pretest, experimental participant underwent the designated treatment for one month. Average score of two-raters was confirmed as final score. Time and amount of treatment was designed as per the capacity of the child.

Bilingualism and language dominance were predictor variables, self-reported through BLP. The dependent variable (morphological ability) was both self-reported and tested through language task. Each session of intervention was of 45 minutes for experimental treatment. Finally, data was compared and analyzed to determine effects of bilingualism and language dominance on morphological ability of language. Contact phenomenon was further studied through 4-M model and the interaction of both languages was observed to identify the frequency and type of morphemes. The 4-M model was used for this purpose in many studies (Wei, 2000; Abu-Rabia \& Sanitsky, 2010; Ali Khan \& Muysken, 2014; Asli-Badarneh \& Leikin, 2019; Birdsong et al., 2012). Findings are presented through inductive qualitative interpretations and quantitative representations, before and after the intervention. Recent models of the bilingual lexicon cater for progressive aspects of delay and reformation of cognitive concepts, including a shared affiliation between L1, L2 and the theoretical dimension (Heine, 2014). Hence, this study has found that inflectional morphology is bound, closed-class and general. English inflectional morphology has two functions in common noun. It marks the common nouns for plurality (number) and possession (genitive case) and both Urdu and English are facilitating Pashto-English that is now in extensive use of Pashto learners.

\section{Conclusion}

The study aimed at identifying factors effecting morphological ability of Pashto-English bilinguals. It is noticed that Pashto mother tongue was strongly maintained by the participant. Bilinguals were able to acquire all four types of morphemes, despite the fact that the language was marginalised and had less representation in the English medium schools. Though Pashto language has dominance in language history score, it has less score in comparison with English on the scale of language proficiency. English proficiency seems ahead of Pashto despite the fact that Pashto maintained a dominant position in language attitude and language use. These results confirm other studies (Saddiqa, 2018; Pipit \& Rahyono, 2020) in bilingual morphological ability but somehow it is different with those where native language is the medium of instruction in schools. The data for this experiment was triangulated through observations, speech notes, and questionnaires. English proficiency was predicted due to extensive exposure in the society, media and schools. Morphological acquisition is the driving force in English as a second language (ESL) development (Dyson, 2009; Schwartz \& Asli, 2014). These two cases confirmed that educational intervention for Pashto early year learners exposed areas of difficulty and then it was inductively analysed through 4M model. Experimental child was observed and it was identified that child was able to communicate well, but somehow reading and writing proficiency got better after a four-week intervention. Cross-linguistic effects on productivity, comprehension and proficiency are evidence of high levels of linguistic proficiency among bilinguals (Lowie, 2000). Somehow, we can relate differences between monolingual and bilingual learners with psycholinguistic factors (linguistic complexity) and sociolinguistic factors (context of exposure). Roberts (1995) provide evidence for the statement that educational intervention for bilinguals need to be specifically designed and developed in such circumstances that are taking into account local and national contexts (Al-Ahdal, 2020) Pashto orthography was introduced first time but its similarity with Urdu and Arabic was another advantage that impacted their acquisition and proficiency. After intervention, child was able to use more advanced vocabulary with early and late system morphemes. This data can offer credible and applicable evidence for educational implications.

\section{Limitations}

This study is limited to a small set of participants, in home-based context, identifying morphological ability and bilingual profile. A more detailed study of factors responsible for the 


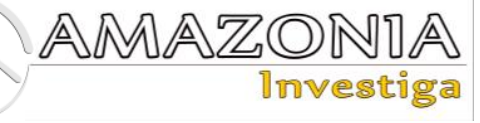

low level of mother tongue and collecting conversation samples of different settings from both mono and bilingual participants (not a testing-only control group) can be very helpful in identifying substantiated factors of the effects of individual cognitive and linguistic differences on morphological ability. Further research including other variables like age, gender, educational level or device of participants on their Language Task Score can be helpful.

\section{References}

Abu-Rabia, S., \& Sanitsky, E. (2010). Advantages of bilinguals over monolinguals in learning a third language. Bilingual Research Journal, 33(2), 173-199.

Al-Ahdal, A. A. M. H. (2020). Translanguagism and the Bilingual EFL Learner of Saudi Arabia: Exploring New Vistas. Asian EFL Journal, 27(1), 14-26.

Ali Khan, A., \& Muysken, P. (2014). Strategies for incorporating nouns and verbs in codemixing: the case of Pashto-English bilingual speech. Lapurdum. Euskal ikerketen aldizkaria| Revue d'études basques| Revista de estudios vascos| Basque studies review, (18), 97-137.

Alnoori, B. S. M. (2019). Writing skills developed through Language Integrated Content. Amazonia Investiga, 8(19), 362-368. https://amazoniainvestiga.info/index.php/amazo nia/article/view/238

Asli-Badarneh, A., \& Leikin, M. (2019). Morphological ability among monolingual and bilingual speakers in early childhood: The case of two Semitic languages. International Journal of Bilingualism, 23(5), 1087-1105.

Beaudrie, S. (2012). A corpus-based study on the misspellings of Spanish heritage learners and their implications for teaching. Linguist. Educ. 23, 135-144. doi: 10.1016/j.linged.2011.09.001

Birdsong, D., Gertken, L. M., \& Amengual, M. (2012). Bilingual language profile: An easy-touse instrument to assess bilingualism. COERLL, University of Texas at Austin.

Borysko, N., Dolyna, A., Bondarenko, E., \& Korniiko, I. (2020). Learning German grammar after English: Let us give Ukrainian students a chance. Amazonia Investiga, 9(29), 516-529. https://amazoniainvestiga.info/index.php/amazo nia/article/view/1416

Bosch, S., Veríssimo, J., \& Clahsen, H. (2019). Inflectional morphology in bilingual language processing: An age-of-acquisition study. Language Acquisition, 26(3), 339-360.

Boussofara-Omar, N. (2013). Revisiting Arabic diglossic switching in light of the MLF and its sub-models: The 4-M Model and the Abstract Level Model. Bilingualism: Language and Cognition, 6 (1), pp. 33-46

Chondrogianni, V., \& Vasić, N. (2016). Crosslinguistic aspects in child L2 acquisition.

International Journal of Bilingualism 2016, 20(4), 361-368.

Coleman, H. (2010). The English language in development. A paper commissioned by the British Council.

Dunn, A. L., \& Tree, J. E. F. (2009). A quick, gradient bilingual dominance scale. Bilingualism: Language and Cognition, 12(3), 273-289.

Dyson, B. (2009). Processability Theory and the role of morphology in English as a second language development: a longitudinal study. Second Language Research, 25(3), 355-376. Gertken, L. M., Amengual, M., \& Birdsong, D. (2014). Assessing language dominance with the bilingual language profile. Measuring L2 proficiency: Perspectives from SLA, 208-225.

Heine, L. (2014). Models of the bilingual lexicon and their theoretical implications for CLIL. The Language Learning Journal, 42(2), 225-237.

Hsu, L. S. J., Ip, K. I., Arredondo, M. M., Tardif, T., \& Kovelman, I. (2019). Simultaneous acquisition of English and Chinese impacts children's reliance on vocabulary, morphological and phonological awareness for reading in English. International journal of bilingual education and bilingualism, 22(2), 207-223.

Itani-Adams, Y., Iwasaki, J., \& Kawaguchi, S. (2017). Similarities and differences between simultaneous and successive bilingual children: Acquisition of Japanese morphology. International Journal of Applied Linguistics and English Literature, 6(7), 268-276.

Kecskes, I. (2006). The dual language model to explain code-switching: A cognitive- pragmatic approach. Intercultural Pragmatics, 3(3), 257-283.

Kecskes, I., \& nde Papp, T. (2000). Foreign language and mother tongue. Psychology Press. Keshavarz, M. H. (2007). Morphological development in the speech of a Persian-English bilingual child. Journal of psycholinguistic research, 36(4), 255-272.

Khan, A. A., \& Khalid, A. (2018). PashtoEnglish codeswitching: Testing the morphosyntactic constraints of the MLF model. Lingua, 201, 78-91.

Khan, S., Akram, W., \& Khan, A. (2016). Functions of Inflectional Morphemes in English and Pashto Languages. Journal of Applied Linguistics and Language Research, 3(1), 197-216. 
López, L. (2018). Toward an Integrated Model of Bilingual Grammar. Chicago, IL: University of Chicago.

Lowie, W. (2000). Cross - linguistic influence on morphology in the bilingual mental lexicon. Studia Linguistica, 54(2), 175-185.

Miikkulainen, R., \& Kiran, S. (2009, June). Modeling the bilingual lexicon of an individual subject. In International Workshop on SelfOrganizing Maps (pp. 191-199). Springer, Berlin, Heidelberg.

Myers-Scotton, C. (2006). Multiple voices: An introduction to bilingualism. USA: WileyBlackwel.

Myers-Scotton, C. M., \& Jake, J. L. (2017). Revisiting the 4-M model: Codeswitching and morpheme election at the abstract level. International Journal of Bilingualism, 21(3), 340-366.

Myers-Scotton, C., \& Jake, J. L. (2000). Testing the 4-M model: An introduction. International journal of bilingualism, 4(1), 1-8.

Nicoladis, E., Palmer, A., \& Marentette, P. (2007). The role of type and token frequency in using past tense morphemes correctly. Developmental Science, 10(2), 237-254.

Nunn, R., \& Adamson, J. (2009). Accepting alternative voices in EFL journal articles. Busan: Asian EFL Journal Press.

Ocal, T., and Ehri, L. (2017b). Spelling pronunciations help college students remember how to spell difficult words. Read. Writ. 30, 947-967. doi: 10.1007/s11145-016-9707-z Pipit, M., \& Rahyono, F. X. (2020). The past tense expression of Indonesian learners: A morphosyntactic review and its implication toward teaching field. Asian EFL Journal, 27(2), 120-143.

Roberts, C. A. (1995). Bilingual education program models: A framework for understanding. Bilingual research journal, 19(3-4), 369-378.
Rock, P. S. (2018). Nuestra escuela: A Quasiexperimental Study of the Impact of Spanish Language Skills Training on the Attitudes and Practices of Non- Hispanic Teachers of Hispanic Students (Doctoral dissertation, Concordia University (Oregon)).

Saddiqa, A. (2018). The Role of Pashto (as L1) and Urdu (as L2) in English Language Learning. Linguistics and Literature Review (LLR), 4(1), 1-17.

Schmid, M. S., \& Köpke, B. (2017). The relevance of first language attrition to theories of bilingual development. Linguistic Approaches to Bilingualism, 7(6), 637-667.

Schwartz, M., \& Asli, A. (2014). Bilingual teachers' language strategies: The case of an Arabic-Hebrew kindergarten in Israel. Teaching and Teacher Education, 38, 22-32.

Snyder, B., \& Barzilay, R. (2008). Unsupervised multilingual learning for morphological segmentation. In Proceedings of acl-08: hlt (pp. 737-745).

Soudi, A., Neumann, G., \& van den Bosch, A. (Eds.) (2007). Arabic computational morphology: Knowledge-based and empirical methods. In Arabic computational morphology (pp. 3-14). Amsterdam, Netherlands: Springer.

van Hooff, L. (2018). A quasi-experimental study into the effects of Bilingualism, Language Dominance, and Language Mindsets on Language Learning Achievement. (Master Thesis, Tilberg University, Netherlands).

Vihman, V. A. (2018). Language Interaction in Emergent Grammars: Morphology and Word Order in Bilingual Children's Code-Switching. Languages, 3(4), 40.

Wei, L. (2000). Types of morphemes and their implications for second language morpheme acquisition. International Journal of Bilingualism, 4(1), 29-43. 